\title{
Independent Component Analysis for Improved Defect Detection in Guided Wave Monitoring
}

\author{
This paper applies guided wave methods to detect damage in complex industrial \\ pipeline structures using independent component analysis (ICA).
}

\author{
By Jacob Dobson and Peter Cawley
}

\begin{abstract}
Guided wave sensors are widely used in a number of industries and have found particular application in the oil and gas industry for the inspection of pipework. Traditionally this type of sensor was used for one-off inspections, but in recent years there has been a move towards permanent installation of the sensor. This has enabled highly repeatable readings of the same section of pipe, potentially allowing improvements in defect detection and classification. This paper proposes a novel approach using independent component analysis to decompose repeat guided wave signals into constituent independent components. This separates the defect from coherent noise caused by changing environmental conditions, improving detectability. This paper demonstrates independent component analysis applied to guided wave signals from a range of industrial inspection scenarios. The analysis is performed on test data from pipe loops that have been subject to multiple temperature cycles both in undamaged and damaged states. In addition to processing data from experimental damaged conditions, simulated damage signals have been added to "undamaged" experimental data, so enabling multiple different damage scenarios to be investigated. The algorithm has also been used to process guided wave signals from finite
\end{abstract}

Manuscript received February 24, 2015; revised June 18, 2015; accepted June 23, 2015. Date of publication September 15, 2015; date of current version July 15, 2016. This work was supported by BP plc, the U.K. Research Centre in NDE, and the U.K. Engineering and Physical Sciences Research Council.

The authors are with the Department of Mechanical Engineering, Imperial College of Science, Technology and Medicine, London SW7 2AZ, U.K. (e-mail: p.cawley@imperial.ac.uk).

Digital Object Identifier: 10.1109/JPROC.2015.2451218 element simulations of a pipe with distributed shallow general corrosion, within which there is a patch of severe corrosion. In all these scenarios, the independent component analysis algorithm was able to extract the defect signal, rejecting coherent noise.

KEYWORDS | General corrosion in pipes; guided wave monitoring; independent component analysis

\section{INTRODUCTION AND RELATED WORK}

Guided wave sensors are widely used in a number of industries and have found particular application in the oil and gas industry for the inspection of pipework. This type of sensor generates a low-frequency ultrasonic wave $(<100 \mathrm{kHz})$ that travels along the axis of the pipe using the walls as a waveguide. The wave has full volumetric coverage and very low attenuation in steel, making it especially suited for long-range screening applications. It is possible to inspect a large length of pipework with only a small number of sensors; a single sensor can routinely inspect more than $50 \mathrm{~m}$ of pipe from a single location [1]. This coverage comes at the cost of lower sensitivity, with commercial guided wave systems typically sensitive to changes in cross section of around 5\% and above in a single inspection [2]. This type of sensor is therefore usually used in conjunction with another localized, high-accuracy technique such as ultrasonic thickness gauging [3] to do followup inspections of the areas the guided wave sensor has identified as suspect.

There are several commercial guided wave systems for pipework, and in all cases these systems use an array of 
sensors attached to the outside of the pipe [4]-[6]. These sensors operate in pulse-echo mode, exciting a controlled wave that travels through the structure. When this wave reaches a change in the impedance of the structure, caused by a change in the cross section for example, some of the wave will be reflected back and received by the sensor. A trained operator can then assess the shape, amplitude, and mode of these reflections to infer the nature of the feature that caused the reflection. Damage such as corrosion will produce a reflection [7], [8], as will benign pipe features such as welds and supports [9]. There can also be signal components due to the excitation and reception of unwanted modes and imperfect direction control [10]. These additional components introduce a background noise that sets the sensitivity of the technique-typically defects must produce a reflection twice as large as the background noise in order to be detected. These unwanted signals are deterministic and cannot be removed through averaging; for this reason, they are often known as coherent noise [10]. Standards exist to guide the collection and interpretation of guided wave data [11], limiting the impact of coherent noise as far as possible.

In the early stages of development, guided wave sensors were typically used in an inspection configuration. A sensor would be attached to a section of pipe, an inspection made, and then the sensor moved to another location. It is, however, becoming more common to permanently bond the sensor to the pipe and operate the sensor in a monitoring configuration [2]. In guided wave techniques, this trend is partly motivated by high access cost. Pipelines are often buried underground or at elevation, requiring costly digging or scaffolding equipment for access. In many cases, access to the pipe will also have health and safety implications. If the sensor is permanently installed, these access costs need only be incurred once, and an umbilical cord can be run to a safer, more convenient location [2].

The other driver for permanent installation is the potential for improvements in defect detection and classification. Because the sensor is bonded to the pipe in a permanent installation, it is possible to obtain highly repeatable readings of the same section of pipe. If we make an initial inspection of the pipe when it is in a known structural condition, we can use this information to subtract out coherent noise from later measurements. This is the principle of baseline subtraction where the early measurement, the baseline, is subtracted from the later measurement, the reading [12].

This technique is effective only if the change between baseline and reading is a change in the structural condition of the structure. If there is also a change in the environmental conditions surrounding the pipe, this will introduce additional changes that are difficult to distinguish from those caused by structural change. This can partly be dealt with by collecting a large number of baselines under different environmental conditions and selecting the optimal baseline to compare to a reading [13]. However, in an industrial setting, it is often not possible to collect multiple signals under prescribed environmental and structural conditions.

In response to this limitation, the baseline stretch technique was developed, which seeks to compensate for signal changes due to temperature, the most significant environmental change for guided wave signals [12]. When the temperature of a pipe changes, the dimensions of the structure change as does the propagation velocity of the inspection wave [14]. This causes changes in the arrival time of reflections and leads to imperfect cancellation during subtraction. Baseline stretch attempts to compensate for this change through compression or dilation of the signal and can be applied globally or to windowed portions of the signal [13], [15].

This compensation procedure is limited however. The stretch process does not perfectly compensate for temperature, and its performance deteriorates as the temperature difference increases. This stretch procedure also ignores the influence of other environmental effects, which have been found to be significant in guided wave systems [16]. Other researchers have therefore investigated baselinefree methods such as the time reversal method, a technique for defect detection based on reciprocal inspections between sensor pairs [17]. Such methods have obtained good results in the laboratory, for example on Lamb wave inspection of composite plates [17], but their effectiveness on industrial data is still an open question. Others have pursued data-driven approaches that take a pool of ultrasonic signals and look for meaningful trends within the data [18], [19]. These data-driven approaches have been based on established data mining techniques such as support vector machines, neural networks, Singular Value Decomposition (SVD), and Principal Component Analysis [20]. Of these, SVD is the most developed for guided wave systems, with several examples in the literature of SVD being successfully applied to experimental data [16], [21]. In these studies, SVD was able to compensate for real environmental and operational variations and extract a representation of the defect with lower coherent noise than the original signal.

Independent Component Analysis (ICA) is similar to SVD in that it seeks to extract meaningful trends in data. The difference between these techniques is that SVD uses a decorrelation of the data to separate the information, while ICA minimizes the mutual information between groupings. ICA has been used alongside neural networks to identify damaged and undamaged states in vibration data from simple truss structures [22], suggesting it may be advantageous to use it with guided wave systems. The purpose of this paper is to investigate the potential of ICA for processing the signals from permanently installed guided wave systems. This is achieved by applying ICA to signals that are representative of a range of monitoring conditions that might be faced in industry. 
Section II introduces Independent Component Analysis and the implementation used in this paper, as well as the procedure for selecting the damage-sensitive feature. Section III then introduces the guided wave data that will be passed to the ICA algorithm, and Section IV shows the results of this analysis. The signals analyzed in Section IV all relate to discrete echoes from features with small axial extent so echoes are well separated. In Section V, we consider the more challenging case of general corrosion growth within which there is a region of more severe growth. Section VI presents the conclusions of the work and a discussion of the potential of the technique.

\section{ICA METHODOLOGY}

Independent Component Analysis is a statistical technique for revealing the hidden trends and groupings that underlie a set of data. The technique takes a set of multidimensional data and transforms it into components that are as statistically independent as possible [23], [24]. The intention of using ICA on guided wave data is that after applying the transform, one of the independent components will contain data relating to the defect, while most coherent noise will be rejected to other independent components; so giving a clearer representation of the defect. Numerous implementations of ICA are available [25], [26], but the FastICA algorithm [23] is used in this study because it achieves similar performance to other algorithms but at greater speed [27]. A brief overview of the FastICA method is given here, but the interested reader can find full details in [23].

The FastICA method assumes that the input data $\mathbf{X}$ can be described by the model

$$
\mathbf{X}=\mathbf{A S}
$$

where $\mathbf{S}$ is a statistically independent representation of the data and $\mathbf{A}$ is a matrix of scalar values that relates $\mathbf{S}$ to $\mathbf{X}$. In the case of guided wave analysis, $\mathbf{X}$ is an $[m \times n]$ matrix of guided wave signals collected from a sensor. Each of the $m$ rows is a guided wave response signal as a function of time (which can be converted to distance knowing the wave velocity), with each of the $n$ columns containing the amplitude of that signal at a certain time. The signals that are the rows of $\mathbf{S}$ will be the new representation we want in which the defect signal and the coherent noise have been separated into different components. Independent component analysis allows us to find $\mathbf{S}$ by performing the operation $\mathbf{S}=\mathbf{W X}$, where $\mathbf{W}$ is the inverse of the mixing matrix $\mathbf{A}$. To calculate $\mathbf{W}$, the algorithm uses the property that the signals in $\mathbf{S}$ must, by definition, be maximally statistically independent. The algorithm iterates through possible values of $\mathbf{W}$ to find the matrix that minimizes mutual information between the rows of $\mathbf{S}$.
When FastICA is applied to guided wave data, the rows of $\mathbf{S}$ will be guided wave signals containing the reflections from different features, while the columns of $\mathbf{A}$ show the amplitude of these signals across the input data. The columns of $\mathbf{A}$ therefore track the amplitude progression of the rows of $\mathbf{S}$, the components. In this paper, all columns of $\mathbf{A}$ are scaled such that the maximum value in each column is one, with the amplitude of the rows of $\mathbf{S}$ adjusted accordingly. Note that the product of the row of $\mathbf{S}$ and the weighting function $\mathbf{A}$ will give the true amplitude of that component of the signal. How the amplitude is apportioned between $\mathbf{A}$ and $\mathbf{S}$ is a matter of presentational clarity. It is then necessary to know which row(s) of $\mathbf{S}$ contain information about defect growth. Since the defect location is known, we merely select the row that is most similar to the defect reflection response. However, if the method is to be used in practice with unknown defects, it will be necessary to produce an automatic method to distinguish components relating to true defect growth from those relating to random noise or environmental effects. A method based on k-means clustering has been proposed in the literature [21], but so far it has only been proven on step-type defect growth. One potential alternative is change detection using the generalized likelihood ratio, as reported in [28].

\section{DATASETS}

\section{A. Laboratory Experiment}

The first set of data comes from an experiment with a $6 \mathrm{~m}$ length of 8 -in schedule 40 piping that was temperature cycled in the laboratory. The purpose of this experiment was to collect commercial quality guided wave signals from a length of plain pipe experiencing known changes in environmental condition. Since temperature is the main contributor to signal changes in guided wave inspection, this was the condition that was varied. With a sufficient pool of data collected in the undamaged condition, the second stage was to introduce a small point defect and see how this changes the received signal.

The pipe was heated via a resistive heating element that was suspended in the center using sheet metal inserts. In this way, the element is prevented from contacting the pipe wall while the sheet metal inserts produce negligible reflection during the guided wave inspection. A commercial guided wave sensor (gPIMS unit manufactured by Guided Ultrasonics, Ltd.) was then installed on the outside of the pipe, $2 \mathrm{~m}$ from one end as well as seven thermocouples distributed across the outer surface. This assembly was then wrapped in an insulating material. A diagram of this setup is given in Fig. 1.

Using the thermocouples, the heating element and an appropriate feedback control loop the temperature of the pipe was varied. The pipe was heated to $90{ }^{\circ} \mathrm{C}$, held to allow the temperature to equalize throughout the pipe, and then cooled to $30{ }^{\circ} \mathrm{C}$. During this cooling 


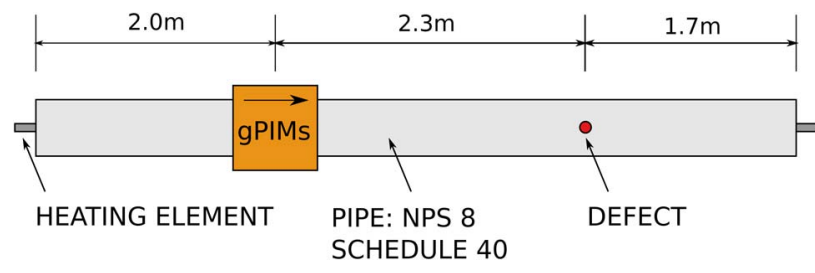

Fig. 1. Equipment for the laboratory experiment: NPS8 pipe with internal heating element and guided wave sensor (gPIMS) permanently attached. Note the noncontacting heating element through the center of the pipe. Not to scale.

cycle, 42 measurements were made using the gPIMS sensor in approximately equal steps of time. The measurements were made using an eight-cycle hanning window toneburst, center frequency $31 \mathrm{kHz}, \mathrm{T}(0,1)$ mode. This process was repeated for six temperature cycles to give a total of 252 measurements across a range of temperatures. An example measurement from the sensor is given in Fig. 2, where only the forward direction is shown (forward direction indicated by arrow in Fig. 1). Note that we consider even those parts of the signal that indicate a distance further than $4 \mathrm{~m}$. These are not caused by physical features beyond the end of the pipe, but arise due to reverberations between the pipe ends. These signal components serve as a useful model for a longer pipe with multiple features, which is how they are used in this study.

After this initial period of temperature cycling, the insulation was removed, and a flat bottom hole was drilled $2.3 \mathrm{~m}$ from the gPIMS sensor. This hole had a depth of $4 \mathrm{~mm}$ and a diameter of $7 \mathrm{~mm}$, representing a cross-sectional change of $0.5 \%$ at its maximum extent. The insulation was then reapplied, and the same process used

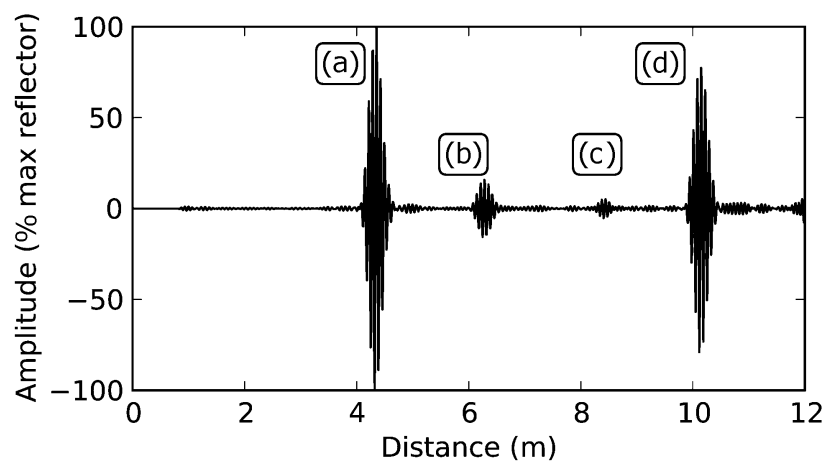

Fig. 2. Example pulse-echo measurement from the laboratory experiment, showing reflected $\mathrm{T}(0,1)$ mode in the forward direction (forward direction indicated by arrow in Fig. 1). Sensor located at the origin, with reflections caused by (a) end reflection, (b) imperfect direction control, (c) ring reflection, and (d) first reverberation. to collect readings from the damaged pipe between $90{ }^{\circ} \mathrm{C}$ and $30{ }^{\circ} \mathrm{C}$.

\section{B. Pipe Loop Experiment}

The second set of data comes from a pipe loop installed at an industrial test site. The purpose of this experiment was to collect commercial quality guided wave signals from a section of pipe with features such as welds, bends, and flanges. The other purpose of the experiment was to understand how these signals change when defects are introduced in both plain sections of pipe and at welds, before and after bends.

Experiments were performed on a section of pipework made from NPS8 schedule 40 piping. At one end, the pipework begins with a blanked flange, connected to a straight section of pipe $5.79 \mathrm{~m}$ long. A weld then joins this to another section of straight pipe that is $6.82 \mathrm{~m}$ long. A Guided Ultrasonics, Ltd., gPIMS unit was permanently attached to this length of pipe, $11.17 \mathrm{~m}$ from the flange. This in turn is connected by a weld to a $90^{\circ}$ bend, beyond which there is a further section of straight pipe that extends beyond the region of interest. The pipe is a closed system filled with a liquid whose temperature can be controlled; in this way, the temperature of the pipe could be controlled during the experiment. This setup is shown in Fig. 3.

Initially, the temperature of the pipe loop was set to $38{ }^{\circ} \mathrm{C}$, and four measurements were made under the nodamage condition. The measurement was made using an eight-cycle hanning window toneburst, center frequency $31 \mathrm{kHz}, \mathrm{T}(0,1)$ mode. The temperature of the loop was then ramped to $90{ }^{\circ} \mathrm{C}$ and allowed to cool again to $38{ }^{\circ} \mathrm{C}$. The purpose of this ramping was to cause thermal stress in the sensor and study the effect of sensor changes due to environmental cycling. Three 1-in-diameter flat bottom holes were then drilled to give defects A, B, and C indicated in Fig. 3. Defect A was drilled in a clean section of pipe and should be the easiest to detect. Defect B was drilled adjacent to a weld and will be more difficult to detect due to masking by the weld reflection. Defect $\mathrm{C}$ is adjacent to a weld and beyond a bend, making it the most difficult to detect due to masking and the fact that bends tend to exacerbate environmental effects. Initially, the holes in these three locations were drilled to a depth that gives a $0.25 \%$ cross-sectional change. Four measurements were made using the sensor, and the loop is again ramped to $90{ }^{\circ} \mathrm{C}$ and

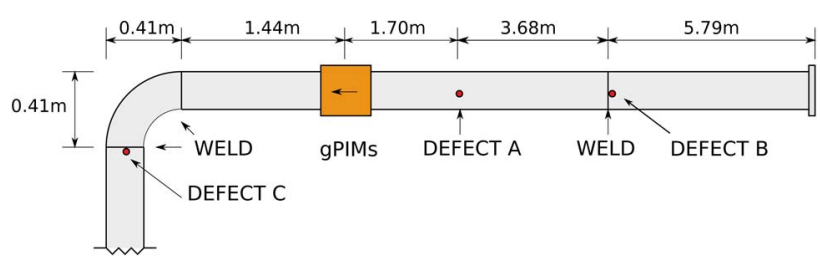

Fig. 3. Equipment for the pipe loop experiment: feature-rich pipe with welds and bends, filled with temperature controlled fluid and with a guided wave sensor (gPIMS) permanently attached. Not to scale. 


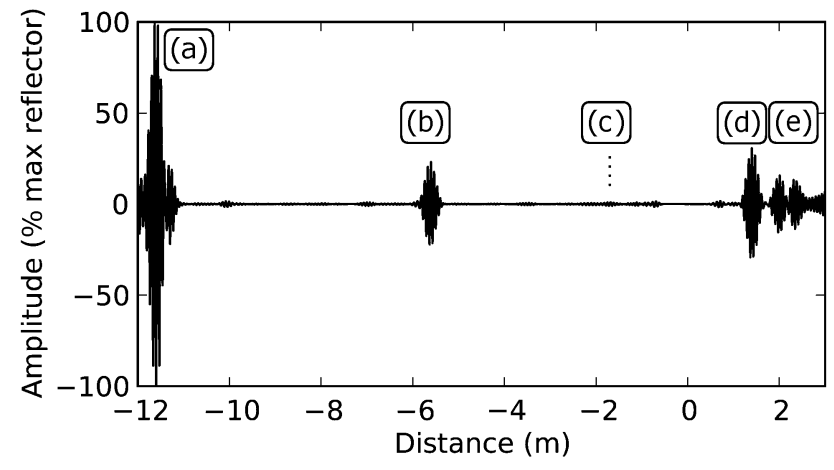

Fig. 4. Example pulse-echo measurement from the pipe loop experiment showing reflected $T(0,1)$ mode in the forward and backward directions (forward indicated by arrow in Fig. 3). Sensor located at the origin $(0 \mathrm{~m})$, with reflections caused by (a) flange, (b) weld and defect $B$, (c) defect $A$, (d) weld before bend, and (e) weld after bend and defect $C$.

cooled to $38{ }^{\circ} \mathrm{C}$. The holes were enlarged to $0.5 \%$ crosssectional change, and another four measurements made. This process was repeated a final time for a hole with $0.75 \%$ cross-sectional loss. An example output from the sensor is given in Fig. 4, with positive direction indicated by the arrow in Fig. 3.

\section{Data Post-Processing}

It is possible to apply ICA directly to the signals from the laboratory and pipe-loop experiments. However, it was found that the ICA performed better if the signals were first partly compensated for environmental changes. For the results presented in this paper, each signal has been compensated using a stretch algorithm. This stretch involves dilation or compression of the signal in the time domain, an amplitude scaling, and a delay, the purpose being to bring the signal as close as possible to a reference signal. This manipulation was performed in the frequency domain and in this study was achieved using a simulated annealing optimization to optimize across the three variables simultaneously. Note that other implementations are available [13], [15]. For both the laboratory experiment and pipe loop experiment, a suitable reference signal had to be chosen to stretch to. This reference was arbitrarily chosen as the signal with a temperature closest to the mean.

\section{RESULTS OF APPLYING ICA}

\section{A. Laboratory Experiment With Simulated Simple Step Defect}

The first test of the algorithm was to see if it could identify a simple step change in a pipe undergoing temperature changes. This scenario was modeled by taking data from the undamaged pipe in the laboratory experiment and adding a signal that models the reflection from a point defect. This first test looked at three scenarios: case A that uses 20 experiment signals collected in the temperature range $60{ }^{\circ} \mathrm{C}-63{ }^{\circ} \mathrm{C}$, case $\mathrm{B}$ that uses 20 signals collected in the temperature range $60{ }^{\circ} \mathrm{C}-70{ }^{\circ} \mathrm{C}$, and case $\mathrm{C}$ that uses 10 signals in the temperature range $60{ }^{\circ} \mathrm{C}-70{ }^{\circ} \mathrm{C}$ and 10 signals in the temperature range $71^{\circ} \mathrm{C}-80^{\circ} \mathrm{C}$. The specific temperatures for each case are given in Table 1. Note that these temperatures do not come directly from thermocouple measurements, but have been inferred from the dilation or compression between signals within a known temperature range. In all three cases, the first 10 signals were left in the undamaged condition, while the second 10 signals had another signal added. This signal is a model of the reflection from a point defect and is simply an amplitude scaled version of the excitation signal used by the sensor: an eight-cycle hanning window toneburst, center frequency $31 \mathrm{kHz}$. This signal was added at a constant amplitude of $0.75 \%$, thus simulating a simple step change in the structural condition of the pipe. The amplitude is given here as a percentage of the amplitude of the largest reflector in the signal (the first end reflection). This signal was added at a distance of $7.1 \mathrm{~m}$ from the transduction, remote from the major feature reflections. Note that this is not a physically possible damage location in the $6 \mathrm{~m}$ pipe, but instead is a portion of the signal that arises due to reverberation between the pipe ends.

Some of the outputs from the ICA algorithm for case A are shown in Fig. 5. Parts (a) and (b) are the signal (row of matrix s) and weighting function (column of matrix $A$ ) for the defect signal, showing a defect signal that is clearly separated from coherent noise and a weighting function that correctly tracks the true amplitude of the defect [dotted line in part (b)]. Parts (c)-(f) show some of the other output signals from the algorithm. Parts (c) and (d) show the signal and weighting for end reverberations and ring reflections, these parts having constant weighting

Table 1 Temperature Distribution of Signals Across Three Example Cases: $A, B$, and $C$

\begin{tabular}{||c||c|c|c|}
\hline \multicolumn{4}{|c|}{ Temperature ${ }^{\circ} \mathrm{C}\left(+/-0.5^{\circ} \mathrm{C}\right)$} \\
\hline Signal Number & Case A & Case B & Case C \\
\hline 1 & 60 & 70 & 70 \\
2 & 60 & 68 & 68 \\
3 & 60 & 60 & 60 \\
4 & 61 & 68 & 68 \\
5 & 61 & 69 & 69 \\
6 & 61 & 61 & 61 \\
7 & 62 & 64 & 64 \\
8 & 62 & 63 & 63 \\
9 & 63 & 68 & 68 \\
10 & 63 & 64 & 62 \\
11 & 60 & 69 & 72 \\
12 & 60 & 62 & 74 \\
13 & 60 & 63 & 71 \\
14 & 61 & 61 & 71 \\
15 & 61 & 61 & 79 \\
16 & 61 & 69 & 80 \\
17 & 62 & 66 & 76 \\
18 & 62 & 65 & 71 \\
19 & 63 & 61 & 72 \\
20 & 63 & 60 & 74 \\
\hline
\end{tabular}



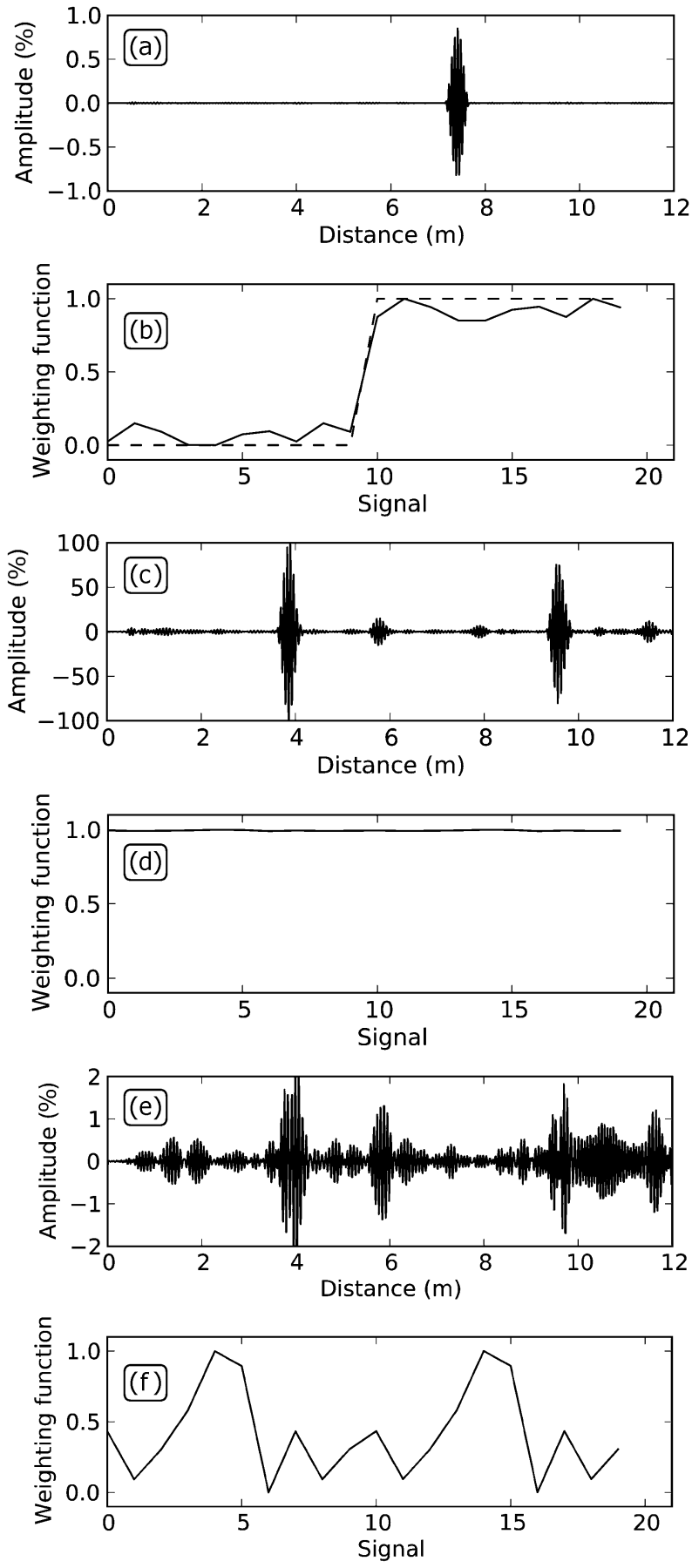

Fig. 5. Output from the ICA algorithm for a simulated step defect in the laboratory experiment (temperature case A), showing: (a) the recovered defect signal and (b) the associated weighting function (solid line is the weighting calculated by ICA, while the dotted line is the true weighting [i.e., the known weighting of the added defect]), (c) the major feature reflections and (d) the associated weighting function, (e) one component of the noise introduced from imperfectly compensated environmental effects and (f) the associated weighting function.
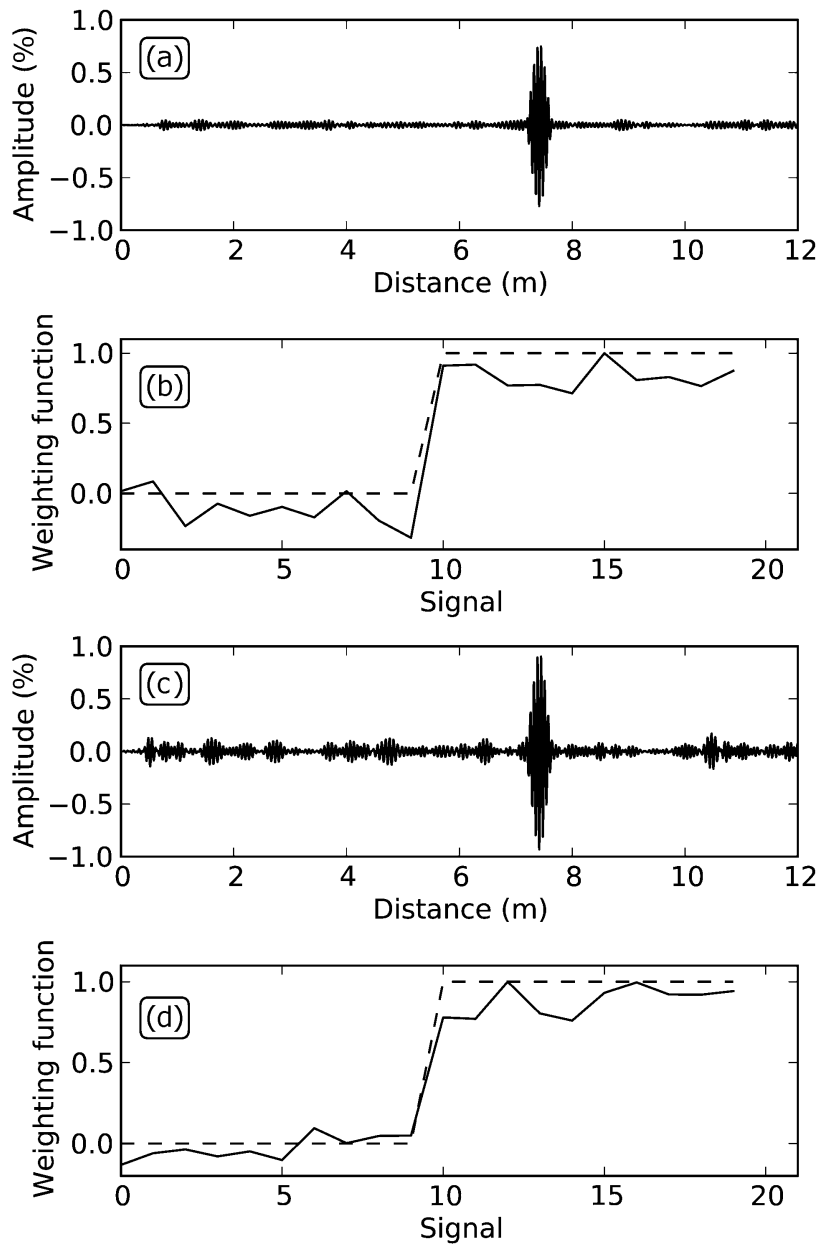

Fig. 6. Example output of the ICA algorithm for simulated step defects in the laboratory experiment, showing: (a) defect signal for temperature case $B$ and (b) the associated weighting function, (c) the defect signal for temperature case $C$ and (d) the associated weighting function.

because these parts of the signal do not change across the input signals. Parts (e) and (f) show the signal and weighting for one of the components of noise introduced by imperfectly compensated environmental effects. The noise component with the largest amplitude has been shown, but the ICA algorithm outputs a total of six noise signals for this set of inputs. These noise signals all have similar oscillating weighting functions, but their maximum amplitude varies from $2 \%$ in the case shown in Fig. 5(e) to a minimum of $0.4 \%$. Although in some cases the noise components have a larger amplitude than the defect signal, their weighting function is different to that of the defect. This difference in weighting function may be one way of distinguishing between damage and noise components.

The ICA output for temperature case B is shown in Fig. 6(a) and (b), where only the defect signal has been shown. The other outputs of the algorithm are similar to those shown in Fig. 5 although there are now 10 noise 
components due to imperfectly compensated environmental variations. These noise components have a maximum amplitude that varies from $4.0 \%$ to $0.2 \%$ across the 10 noise outputs. This increase in the number of components is due to the greater temperature difference among the input signals, which causes an overall increase in the background noise. These noise components all have nonmonotonic weighting functions that vary in a pattern similar to that shown in Fig. 5(f). Similarly, the defect signal for temperature case $\mathrm{B}$ has more coherent noise than temperature case A. This is again thought to be because there is greater temperature difference among the input signals, and hence the algorithm has greater difficulty separating the defect signal. The ICA output for temperature case $\mathrm{C}$ is shown in Fig. 6(c) and (d), where only the defect signal has been shown. As before, the other outputs of the algorithm are similar to those shown in Fig. 5, although there are now 14 noise components in the ICA output. These noise components have a maximum amplitude that varies from $6.0 \%$ to $0.1 \%$, with the maximum noise occurring at the end reflections. Again, the weighting functions for these noise components are nonmonotonic, varying in patterns similar to those shown in Fig. 5(f). The noise on the defect signal is also higher than temperature case A or B, again because there is greater temperature difference on the input signals. It is likely that greater temperature variations would lead to increased noise and deteriorating performance of the ICA, and this is now being investigated.

\section{B. Laboratory Experiment With Simulated Growth of a Flat Bottom Hole}

The second test of the algorithm was to look at a more realistic model for defect growth. Instead of modeling a simple step change, this test modeled the reflection from a flat bottom hole with increasing cross-sectional loss over time, but a constant depth-to-diameter ratio. This is a model of, for example, growing localized corrosion. This scenario was modeled by taking 20 signals from the undamaged pipe in the laboratory experiment and adding a defect signal to the final 10 . For the sake of brevity, only temperature case $\mathrm{B}$ is presented in this paper, where all signals have a temperature within the range $60{ }^{\circ} \mathrm{C}-70{ }^{\circ} \mathrm{C}$ (see Table 1 for details). The added defect signals were generated using the analytical model of Cegla et al. [29]. Using this model the reflection coefficient versus frequency behavior of 10 different flat bottom holes was calculated. Each hole had a depth-to-diameter ratio of 1:2 and represented a cross-sectional loss of between $0.1 \%$ and $1.0 \%$ in the 8 -in pipe. These reflection coefficient curves are shown in Fig. 7. To generate a signal to add to the laboratory data, the frequency spectrum of the inspection signal (eightcycle hanning window toneburst, $31 \mathrm{kHz}$ center frequency) was multiplied by these curves. Transforming this data back into the time domain gave a signal with the correct amplitude and frequency content for a reflection from each hole. Note that as the hole size increases, the frequency

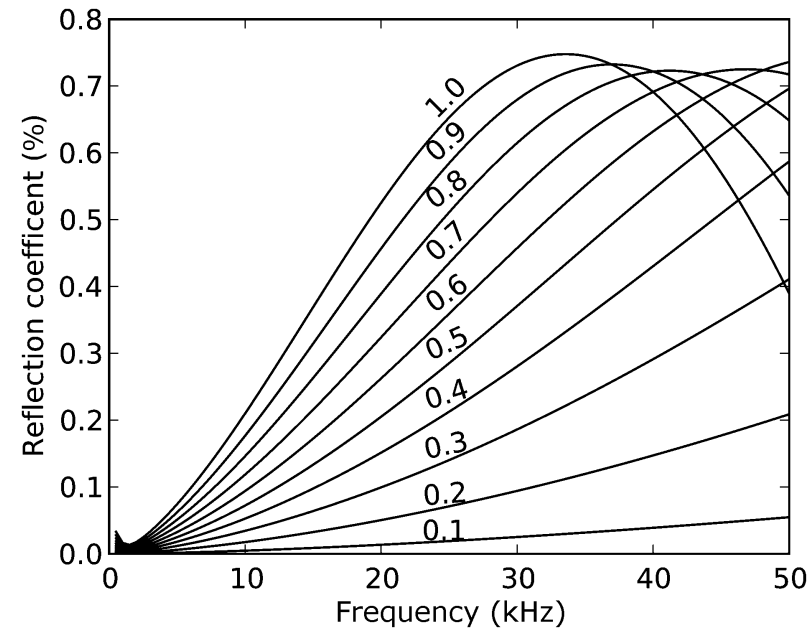

Fig. 7. Reflection coefficient versus frequency behavior for a flat bottom hole. Values along each curve are the percentage cross-sectional change represented by each curve. All holes have a constant depth-to-diameter ratio of 1:2.

content of reflection changes, so the defect signal is no longer simply an increasing amplitude of the same signal. It is of interest to see whether ICA can still identify this hole as a single component at all hole sizes. Although this model is for flat bottom holes in plates, the ratio of pipe radius to hole radius was large enough to justify this approximation. This signal was again added at a distance of $7.1 \mathrm{~m}$, away from major feature reflections.

The results from the ICA algorithm are given in Fig. 8, which shows the defect signal and associated weighting function. The figure shows that the reflection from the
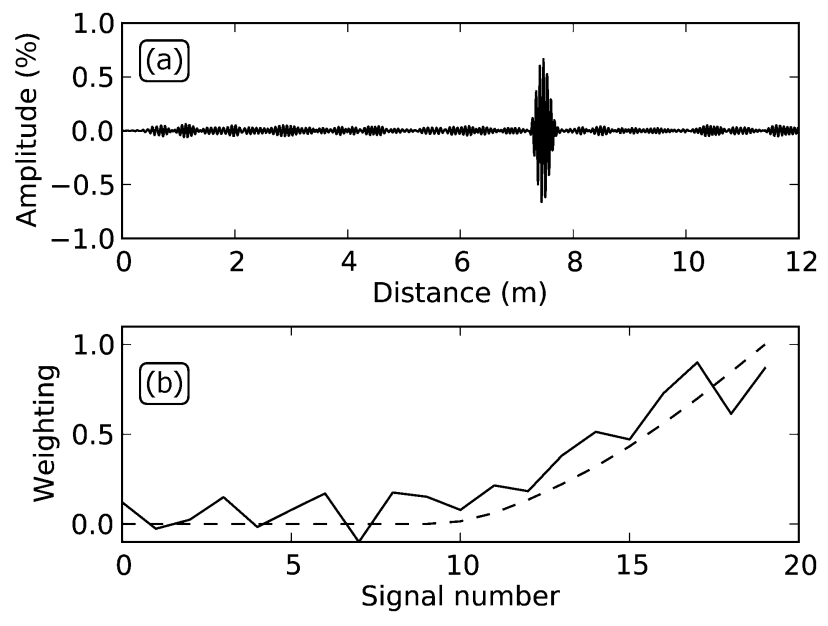

Fig. 8. Output from the ICA algorithm for a simulated flat bottom hole grown in stages in the laboratory experiment data, showing: (a) defect signal and (b) the associated weighting function. Solid line is recovered weighting function, while dotted line is the true. Signals have the temperature variation described by case $B$. 
defect can be identified and the weighting function correctly tracks the true weighting function [dotted line in Fig. 7(b)], albeit with some noise. The other outputs from the algorithm are similar to those shown in Fig. 5, with a total of 10 noise outputs in this case. The maximum amplitude of these noise components varies from 3.7\% to $0.4 \%$, and their weighting functions vary nonmonotonically in a pattern similar to Fig. 5(f).

\section{Laboratory Experiment With Drilled Flat Bottom Hole}

The previous tests with laboratory data have used signals from the undamaged pipe with added artificial defect signals. This is a reasonable approach given that the interaction of guided waves with defects in pipes is well understood [29]. However, there may be some subtlety that arises with a physically introduced defect that is not captured in this previous approach. For that reason, the next test of the ICA algorithm involved data from both the undamaged and damaged pipe in the laboratory experiments. Thirty signals were selected, 15 from a pipe in the undamaged condition and 15 from a pipe containing a 7-mm-diameter, 4-mmdeep flat bottom hole; such a hole represents a crosssectional loss of $0.75 \%$ This hole was drilled $2.3 \mathrm{~m}$ from the sensor. These 30 signals were collected in the temperature range $60{ }^{\circ} \mathrm{C}-70{ }^{\circ} \mathrm{C}$, with the temperatures covering this full range. These signals were then passed to the ICA algorithm, although note that only the first $4 \mathrm{~m}$ of the signals were processed to keep the analysis physical.

The results of the ICA algorithm are given in Fig. 9, which shows: (a) the reflection from the flat bottom hole (at a distance of $2.3 \mathrm{~m}$ ), and (b) the associated weighting function. The algorithm has extracted the defect signal with a fairly low level of coherent noise given the size of the
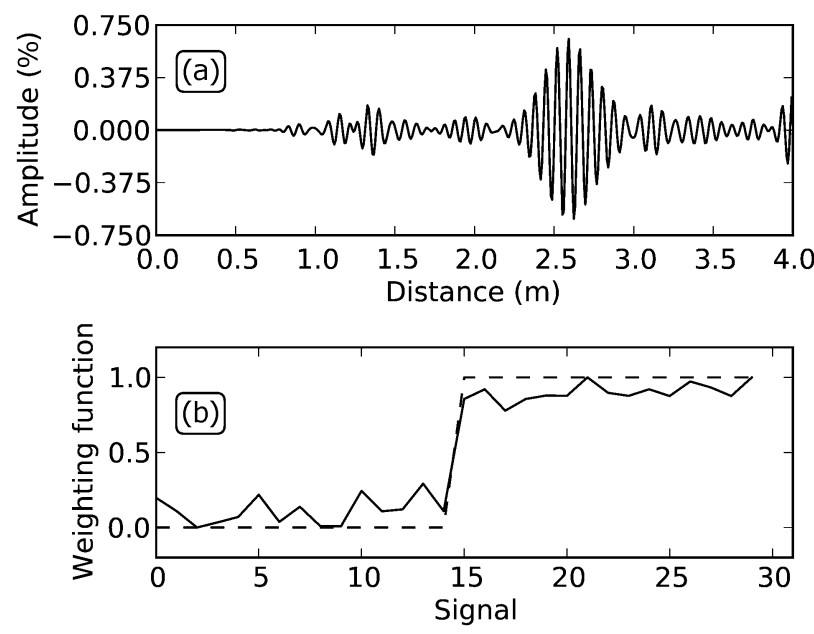

Fig. 9. Result of applying the ICA algorithm for a drilled flat bottom hole in the laboratory experiments, showing: (a) the reflection from the flat bottom hole, beginning at $2.4 \mathrm{~m}$, and (b) the associated weighting function (dotted line is true). All signals at $60^{\circ} \mathrm{C}-70^{\circ} \mathrm{C}$. defect and has accurately tracked the amplitude growth of the signal. Note that in this case the algorithm is trying to extract a defect reflection of around $0.75 \%$, whereas in one off guided wave nondestructive testing, the operator would typically look to detect changes of $5 \%$ or greater. The other outputs of the ICA algorithm are similar to those shown in Fig. 5, with a total of 12 noise components. These noise components have a maximum amplitude that varies from $1.4 \%$ to $0.2 \%$, and again the corresponding weighting functions vary nonmonotonically in a pattern similar to Fig. 5(f).

\section{Pipe Loop Experiment With Physical Flat Bottom Holes}

The last analysis on signals with discrete echoes looks at the reflection from holes physically introduced in the pipe loop experiment. As with the previous test, the defects in this case have been physically drilled in the pipe. The difference compared to the previous case is that these holes have been grown in stages and have been drilled not only in clean sections of pipe, but also close to welds and beyond bends. Fourteen signals were selected, with this group representing four different damage conditions: four signals in the undamaged condition, four signals with holes representing a $0.25 \%$ cross-sectional change, four signals with holes representing a $0.50 \%$ cross-sectional change, and three signals representing a $0.75 \%$ cross-sectional change. All of these signals were collected at a temperature of $38^{\circ} \mathrm{C}$, but with temperature cycling in between each damage condition to simulate ageing of the sensor and hence possible changes in sensitivity or frequency response.

The analysis of this data is split into two: the data from the "forward" direction looking towards the bend and the data in the "backwards" direction that looks towards the flange (forward direction indicated by arrow in Fig. 3). Looking in the backwards direction first [Fig. 10(a) and (b)], the ICA algorithm extracts defects A and B, which are in a clean section of pipe and close to the weld, respectively. Both defects occur on the same output because the holes were drilled at the same time. All signals therefore either have neither or both defects present. Although the defects have the same cross-sectional loss, they have different amplitudes in the output from the ICA. It is thought that the amplitude of defect $B$ is underestimated because of the difficulty in separating it from the reflection from the neighboring weld. The weighting function [Fig. 10(b)] correctly tracks the known weighting function of the defect. The other outputs from the ICA algorithm are the unchanging weld and flange reflections and 12 noise components. These noise components have a maximum amplitude between $4 \%$ and $0.2 \%$ and weighting functions that vary nonmonotonically in patterns similar to Fig. 5(f). In the forward direction [Fig. 10(c) and (d)], the algorithm extracts the reflection from the drilled hole with little coherent noise. The algorithm also correctly tracks the weighting of the defect, albeit underestimating the amplitude when the defect is small. The fact that the weighting 

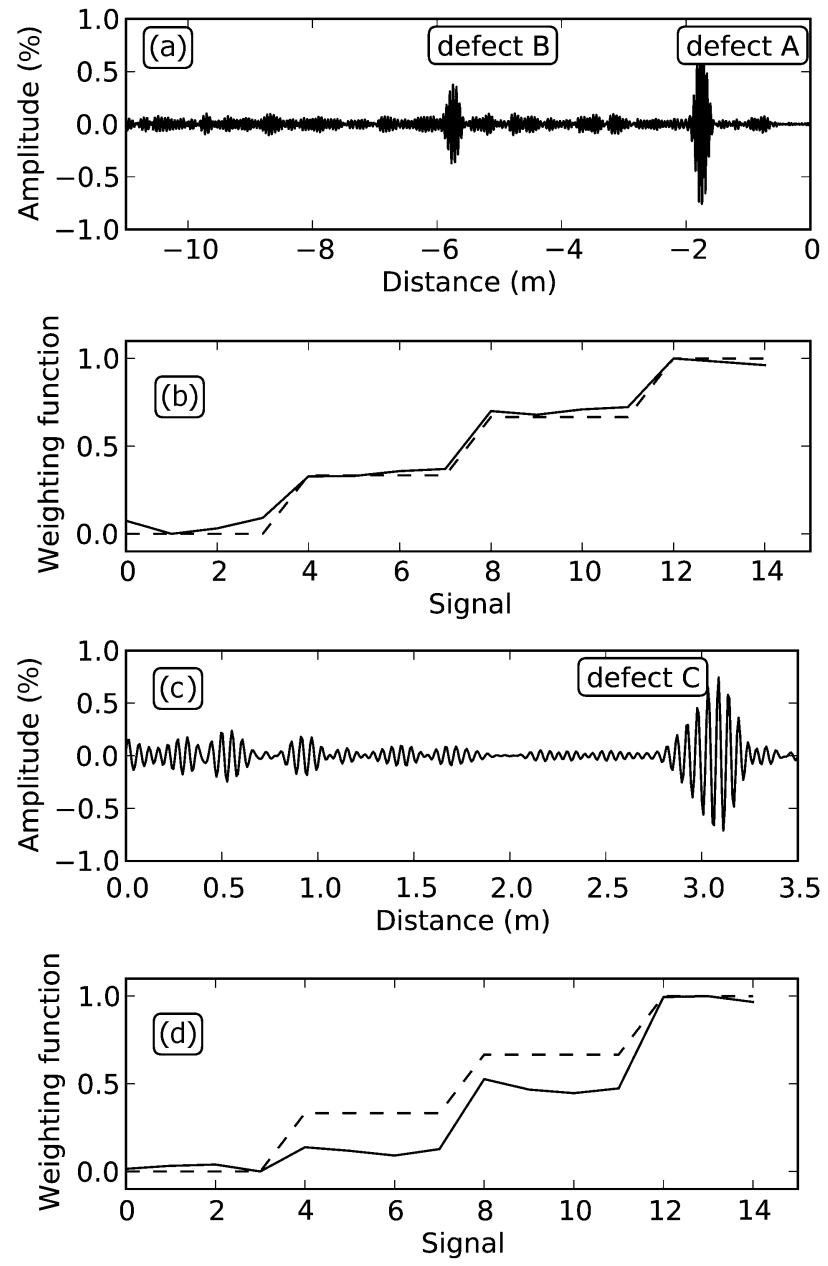

Fig. 10. Results of applying ICA to pipe loop data showing: (a) defect A and $B$, (b) the associated weighting function, (c) defect $C$, and (d) the associated weighting function. All signals collected at $38^{\circ} \mathrm{C}$.

function is poorer in the forward direction than the backwards direction is to be expected: A defect at a weld beyond a bend is a challenging inspection scenario, especially for this size of defect. The other outputs from the ICA algorithm are the unchanging reflection from the welds before and after the bend and 12 noise components. These noise components have a maximum amplitude between $4 \%$ and $0.2 \%$ and weighting functions that vary nonmonotonically in a pattern similar to Fig. 5(f).

\section{SIMULATED GENERAL CORROSION}

\section{A. Method}

The analysis so far has focused on discrete echoes from features with small axial extent. The purpose of this section is to consider the case of interacting echoes from distributed general corrosion. Specifically, we are interested in whether the ICA algorithm is able to identify the reflection from a region of deep corrosion growing within a patch of shallower corrosion. Finite element simulations were used to study this issue given the difficulty of preparing long sections of pipe with a prescribed surface profile.

The finite element model consisted of a 3.0-m-long section of pipe, $168-\mathrm{mm}$ outer diameter, and a wall thickness of $7 \mathrm{~mm}$ (NPS 6 schedule 40). This model was discretized into tetrahedral elements with a characteristic length of $2 \mathrm{~mm}$ using the Netgen algorithm [30]. The elements were then given the properties of steel except for the first $0.3 \mathrm{~m}$ that was set up as an absorbing region following Petit et al. [31]. This absorbing region attenuates all waves entering it to negligible amplitude. The inner surface of the pipe from 0.7 to $2.7 \mathrm{~m}$ was then adjusted to follow a given corrosion profile. The corrosion profile was generated separately as a grid $2.0 \mathrm{~m}$ by $0.484 \mathrm{~m}$ (inner circumference of the pipe) broken into 2-mm elements. Each point in the grid was given a value taken from a Gaussian distribution with desired mean and given standard deviation. An analysis of industrial corrosion patches indicates that the Gaussian distribution is a suitable model for distributed general corrosion [32]. This surface was then multiplied by a 3D-Gaussian to give the surface a specific correlation length in both directions, and all heights greater than zero are set to zero (no increase in pipe thickness). The nodes of the pipe model were then adjusted to match this corrosion profile, with smoothing applied in the radial direction to reduce mesh distortion. Further mesh smoothing was applied using the mesh smoothing function in Gmsh [33].

A uniform tangential force was then applied to a ring of nodes on the outside surface of the pipe, $0.4 \mathrm{~m}$ from one end. This changing force generated a $\mathrm{T}(0,1)$ mode, eightcycle hanning window toneburst, center frequency $31 \mathrm{kHz}$. This model was then stepped in time using POGO, a finite element solver based on the GPU [34]. This software is two orders of magnitude faster than similar commercial packages and enables the current work [34]. The $\mathrm{T}(0,1)$ mode reflection from the corrosion patch was then measured at the location where the $\mathrm{T}(0,1)$ mode was generated. This model is shown in Fig. 11.

\section{B. Dataset}

Initially, 10 random corrosion surfaces were generated having a correlation length of $5 \mathrm{~mm}$ and a root mean square depth of $0.1 \mathrm{~mm}$. These shallow surfaces were used to generate a low level of background noise to model the low levels of coherent noise observed when inspecting a nominally clean section of pipe. A further 10 surfaces were

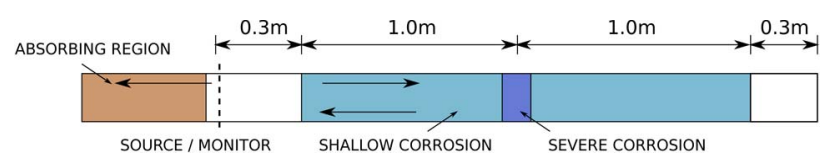

Fig. 11. Geometry of the model used in general corrosion simulations. Not to scale. 
then generated to model a patch of general corrosion gradually increasing in depth and severity.

The first surface was a Gaussian random surface with a correlation length of $5 \mathrm{~mm}$ and a root mean square depth of $0.15 \mathrm{~mm}$. However, a small region of this surface was given a root mean square depth of $0.3 \mathrm{~mm}$. This region extended from 0.95 to $1.05 \mathrm{~m}$ along the axis of the pipe and around half of the circumference, with Gaussian smoothing applied at the interface with the rest of the corrosion surface to prevent discontinuities.

To generate the second surface, a perturbing surface was then added to this original surface. The perturbing surface had an increased correlation length and root mean square depth and again had a small region from 0.95 to $1.05 \mathrm{~m}$ where the corrosion was deeper. This perturbing surface used a random number grid that was a 50:50 weighting of the previous random number grid and a new random number grid. This process of taking the previous surface and perturbing it was continued nine times to give a total of 10 random surfaces with increasing mean depth and correlation length. The correlation length of the perturbing surface was increased from 5 to $50 \mathrm{~mm}$, and the mean depth was increased from 0.1 to $0.5 \mathrm{~mm}$ across the nine surfaces. This gave the final corrosion surface shown in Fig. 12, where the shallow general corrosion had a maximum depth of $1.5 \mathrm{~mm}$, while the deep corrosion pits had a maximum depth of $3 \mathrm{~mm}$. Note that these are absolute limits on the depth; outlier regions deeper than this have been set to the maximum depth. Although convoluted, this process of perturbing previous surfaces was preferable to generating completely new random surfaces for each increment. This is because a perturbation more closely resembles the physical corrosion process. In the real corrosion of pipework, each new corrosion surface must necessarily be an increment of the corrosion surface that preceded it.

The amplitude of $\mathrm{T}(0,1)$ reflection from the general corrosion surface in Fig. 12 is shown as the solid black line in Fig. 13. This reflection amplitude is shown alongside the cross-sectional loss represented by the corrosion. As ex-

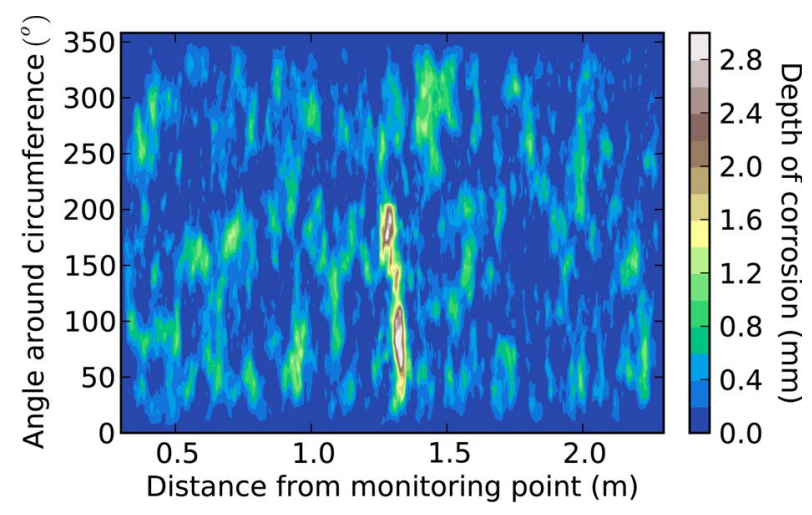

Fig. 12. Thickness map of the final state of the simulated corrosion patch.

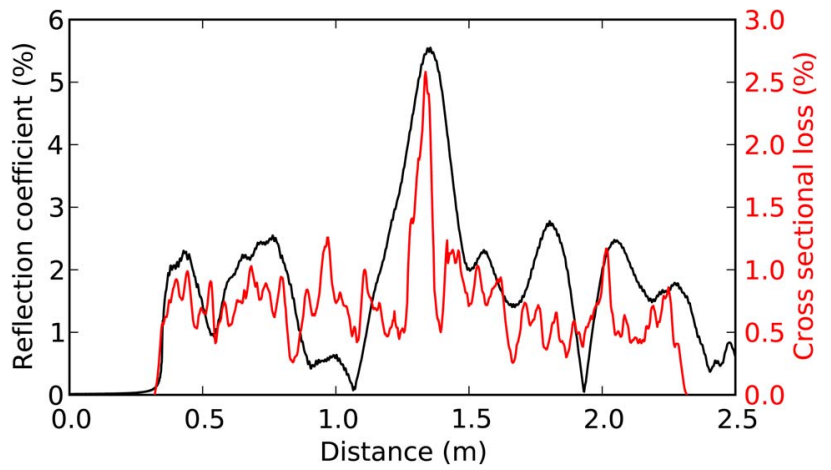

Fig. 13. Overlay of cross-sectional loss and reflection coefficient for the corrosion patch. Note that the corroded surface starts at a distance of $0.3 \mathrm{~m}$ from the monitoring point.

pected, there is broad agreement between the two variables, although the agreement will not be perfect due to the complex constructive and destructive interference between overlapping waves. This figure shows clearly the increased reflection from the more severely corroded section of pipe. Note that this figure does not show the reflection from the end of the pipe ( $2.6 \mathrm{~m}$ from the monitoring point) that dominates the received signal.

\section{Results of Applying ICA Algorithm}

Some example outputs from the ICA algorithm are given in Fig. 14, where the input to the ICA algorithm is the first $3.0 \mathrm{~m}$ of the signals from the model. Fig. 14(a) and (b) shows the signal and weighting function for the severely corroded section of pipe. This signal contains a major reflection from the severely corroded region and is the only signal of this type in the ICA output. This is a very encouraging result, showing that ICA tracks the severe corrosion, keeping it in single component even though the corrosion is growing at more than one point and its shape is changing. Fig. 14(c) and (d) shows the signal and weighting function for the pipe end reflection. This weighting function correctly shows a decrease in the amplitude of the reflection across the final 10 signals as the corrosion grows. This happens because the increase in corrosion causes a reduction in the $\mathrm{T}(0,1)$ mode reaching the pipe end. Fig. 14(e) and (f) shows the signal and weighting function for one of the signal components due to the shallow general corrosion. Note that the algorithm separates the reflection from the shallow corrosion into 14 components, with the largest amplitude component shown in Fig. 14. The other components have a maximum amplitude from $2.4 \%$ to $0.6 \%$, and these components constructively and destructively interfere to give the total reflection seen in Fig. 13.

\section{CONCLUSION}

In this paper, we have discussed the use of Independent Component Analysis for improved defect detection in guided 

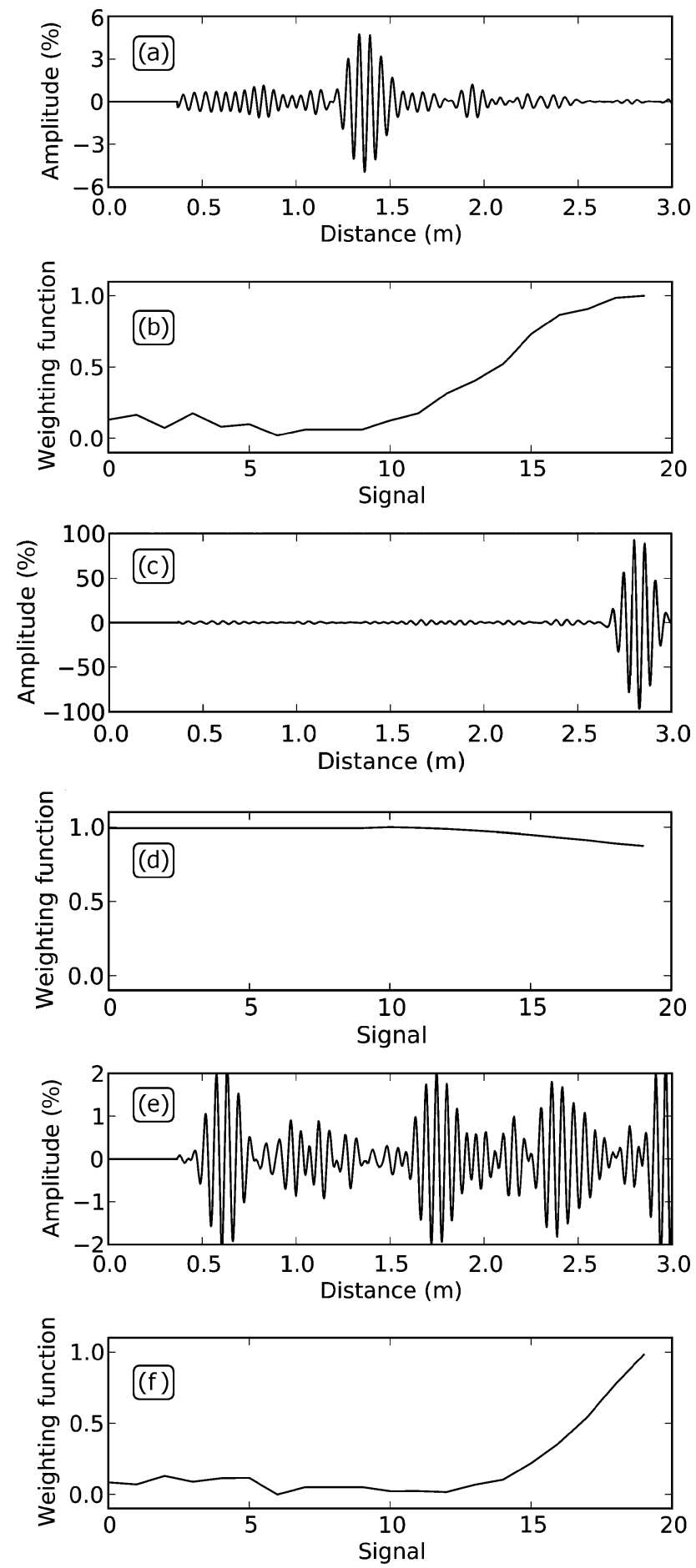

Fig. 14. Results of applying ICA to the general corrosion signals, showing: (a) the reflection from the severely corroded region and (b) the associated weighting function, (c) the end reflection and (d) the associated weighting function, and (e) one of the components of the general shallow corrosion and (f) the associated weighting function.

wave monitoring. Through a combination of the traditional baseline stretch approach and the novel use of ICA, we are able to extract a representation of the defect that has lower coherent noise than the original signal. The algorithm can also be used to accurately track the amplitude history of the defect and hence understand its growth pattern. This is in the context of changing environmental conditions.

This paper has looked at experimental data from both a plain section of pipe and a pipe loop containing welds, bends, and flanges. Data were collected from these pipes while they were in their undamaged and damaged condition and undergoing temperature variations. Initially model defect signals were added to the data from the undamaged plain pipe to simulate step changes and gradual defect growth. The ICA algorithm was able to separate these defect signals from coherent noise in the context of changes in temperature and changing frequency content of the defect signal. The ICA algorithm was then applied to data from the undamaged and damaged pipes, and again the algorithm was able to separate the defect signal from coherent noise. This was for physically introduced defects in clean sections of pipe, at welds, and at welds beyond bends. This paper also used finite element simulations to investigate generally corroded sections of pipe with a patch of severe corrosion. It was found that the ICA algorithm correctly separated the reflection due to the severe corrosion and recognized it as a single evolving defect. This is in the context of the corrosion undergoing a complex growth pattern where its shape and amplitude is changing.

It therefore appears that ICA is a promising method for the automated processing of guided wave data. The next stage is to look at ways of automating identification of the component containing information about the defect. The ICA algorithm outputs several components, some of which are noise and some of which contain useful information. A method must be developed for automatically identifying the components containing useful information, and work is in progress on this issue. One promising approach is to use the weighting functions of the components; in all the cases reported here, the noise components have strongly nonmonotonic weighting functions, while the defect signals have near-monotonic weighting functions. The ICA technique is also only one of several possible techniques for processing guided wave monitoring data. A quantitative comparison of the different techniques would indicate when each method is most appropriate. Such a comparison is in preparation and will be reported in the literature shortly.

\section{Acknowledgment}

The authors are grateful to the UK Engineering and Physical Sciences Research Council for funding an Engineering Doctorate studentship for J. Dobson, and to BP for supporting the project and providing access to the test loop. The authors are also grateful to Dr. A. Galvagni for collecting test data, and Guided Ultrasonics, Ltd., for providing the equipment and assistance in performing the laboratory experiments. Supporting data is available on request: please contact me-ndtgroup-dl@imperial.ac.uk. 


\section{REFERENCES}

[1] M. Lowe and P. Cawley, "Long range guided wave inspection usage - Current commercial capabilities and research directions, Imperial College London, Tech. Rep. [Online]. Available: http://www3.imperial.ac.uk/pls/ portallive/docs/1/55745699.PDF

[2] P. Cawley, F. Cegla, and A. Galvagni, "Guided waves for NDT and permanently-installed monitoring," INSIGHT, vol. 54, pp. 594-601, 2012.

[3] P. Cawley, F. Cegla, and M. Stone, "Corrosion monitoring strategies - Choice between area and point measurements," J. Nondestructive Eval., vol. 32, pp. 156-163, Jun. 2013.

[4] Guided Ultrasonics, Ltd. [Online]. Available: http://www.guided-ultrasonics.com

[5] Plant Integrity, Ltd., "Teletest focus," Jan. 2015. [Online]. Available: http://www. plantintegrity.com/teletest/teletest-focus/

[6] Guided Wave Analysis LLC. [Online]. Available: http://www.gwanalysis.com

[7] R. Carandente and P. Cawley, "The scattering of the fundamental torsional mode from axi-symmetric defects with varying depth profiles in pipes," J. Acoust. Soc. Amer., vol. 127, pp. 3440-3448, Jul. 2010.

[8] A. Lovstad and P. Cawley, "The reflection of the fundamental torsional guided wave from multiple circular holes in pipes," NDT \& E Int., vol. 44, pp. 553-562, Nov. 2011

[9] A. Galvagni and P. Cawley, "The reflection of guided waves from simple supports in pipes," J. Acoust. Soc. Amer., vol. 129, pp. 1869-1880, 2011.

[10] D. Thompson and D. Chimenti, Eds., Practical Long Range Guided Wave Inspection-Managing Complexity, vol. 657. College Park, MD, USA: American Institute of Physics, 2003.

[11] D. Alleyne and A. Demma, "Guided waves: Standardization and certification," in Proc. 6th Int. Conf. Certification Stand. NDT, Jun. 2011, pp. 27-32.

[12] A. Croxford, P. Wilcox, and B. Drinkwater, "Strategies for guided-wave structural health monitoring," Proc. Royal Soc. A, vol. 463, pp. 2961-2981, Aug. 2007.

[13] Y. Lu and J. Michaels, "A methodology for structural health monitoring with diffuse ultrasonic waves," Ultrasonics, vol. 43, pp. 717-731, Oct. 2005.
[14] R. Weaver and O. Lobkis, "Temperature dependence of diffuse phase fields," Ultrasonics, vol. 38, pp. 491-494, Mar. 2000.

[15] T. Clarke, F. Simonetti, and P. Cawley, "Guided wave health monitoring of complex structures by sparse array systems: Influence of temperature changes on performance," J. Sound Vibration, vol. 329, pp. 2306-2322, Jun. 2010.

[16] C. Liu, J. Harley, M. Berges, D. Greve, and I. Oppenheim, "Robust ultrasonic damage detection under complex environmental conditions using singular value decomposition," Ultrasonics, vol. 58, pp. 75-86, Apr. 2015.

[17] H. Park, H. Sohn, K. Law, and C. Farrar, "Time reversal active sensing for health monitoring of a composite plate," J. Sound Vibration, vol. 302, pp. 50-66, Apr. 2007.

[18] Y. Lu and J. Michaels, "Feature extraction and sensor fusion for ultrasonic structural health monitoring under changing environmental conditions," IEEE Sensors J., vol. 9, no. 11, pp. 1462-1471, Nov. 2009.

[19] C. Haynes, M. Todd, E. Flynn, and A. Croxford, "Statistically-based damage detection in geometrically-complex structures using ultrasonic interrogation," Structural Health Monitoring, vol. 12, pp. 141-152, Mar. 2013.

[20] D. Tibaduiza, L. Mujica, and J. Rodellar, "Damage classification in structural health monitoring using principal component analysis and self-organizing maps," Structural Control Health Monitoring, vol. 20, no. 10, pp. 1303-1316, Oct. 2013.

[21] C. Liu et al., "Singular value decomposition for novelty detection in ultrasonic pipe monitoring," in Proc. Sensors Smart Structures Technol. Civil, Mech., Aerosp. Syst., J. P. Lynch, C.-B. Yun, and K.-W. Wang, Eds., San Diego, CA, USA, 2013, vol. 8692.

[22] C. Zang, M. Friswell, and M. Imregun, "Structural damage detection using independent component analysis," Structura Health Monitoring, vol. 3, no. 1, pp. 69-83, Mar. 2004.

[23] A. Hyvarinen, "Fast and robust fixed-point algorithms for independent component analysis," IEEE Trans. Neural Netw., vol. 10, no. 3, pp. 626-634, May 1999.
[24] A. Hyvarinen, J. Karhunen, and E. Oja, Independent Component Analysis. New York, NY, USA: Wiley Interscience, 2001.

[25] A. Delrome and S. Makeig, "EEGLAB: An open source toolbox for analysis of single analysis EEG dynamics including independent component analysis," J. Neurosci. Methods, vol. 134, pp. 9-21, 2004.

[26] P. Hojen-Sorenson, O. Winther, and L. K. Hansen, "Mean field approaches to Independent Component Analysis," Neural Comput., vol. 134, pp. 889-918, Jan. 2002.

[27] D. Langlois, S. Chartier, and D. Gosselein, "An introduction to Independent Component Analysis: InfoMax and FastICA algorithms," Tutorials Quantitative Methods Psychol., vol. 6, pp. 31-38, 2010.

[28] A. Galvagni and P. Cawley, "Reliable identification of damage growth using guided wave SHM systems," in Proc. 7th Eur. Workshop Structural Health Monitoring, Nante, France, 2014, pp. 1720-1727.

[29] F. Cegla, A. Rohde, and M. Veidt, "Analytical prediction and experimental measurement for mode conversion and scattering of plate waves at non-symmetric circular blind holes in isotropic plates," Wave Motion, vol. 45, pp. 162-177, May 2008

[30] J. Schoberl, "Netgen, an advancing front 2D/3D-mesh generator based on abstract rules," Comput. Visual. Sci., vol. 1, pp. 41-52, 1997.

[31] J. Pettit, A. Walker, P. Cawley, and M. Lowe, "A stiffness reduction method for efficient absorption of waves at boundaries for use in commercial finite element codes," Ultrasonics, pp. 1868-1879, 2014.

[32] M. Stone, "Wall thickness distributions for steels in corrosive environments and determination of suitable statistical analysis methods," in Proc. 4th Eur.-Amer. Workshop Rel. NDE, 2009, pp. 1-23.

[33] C. Geuzaine and J. F. Remacle, "Gmsh: A three-dimensional finite element mesh generator with built-in pre- and post-processing facilities," Int. J. Numer. Methods Eng., vol. 79, pp. 1309-1331, 2009.

[34] P. Huthwaite, "Accelerated finite element elastodynamic simulations using the GPU," J. Comput. Phys., vol. 257, pt. A, pp. 687-707, 2014.

\section{ABOUT THE AUTHORS}

Jacob Dobson received the M.Eng. degree in mechanical engineering from Imperial College London, London, U.K., in 2012, and is currently pursuing the engineering doctorate in non-destructive evaluation at the UK Research Centre in NDE (RCNDE), Imperial College London.

His research interests are in guided waves for structural health monitoring and advanced signal processing techniques thereof.

Peter Cawley received the B.Sc. and Ph.D. degrees in mechanical engineering from the University of Bristol, Bristol, U.K., in 1975 and 1979, respectively.

He worked in industry from 1979 to 1981 and then joined the Mechanical Engineering Department, Imperial College London, London, U.K., initially as a Lecturer and then successively Senior Lecturer, Reader, and Professor. He is now head of the Imperial College Mechanical Engineering Department and leads the NDE research group; he is also the principal investigator of the UK Research Centre for NDE (RCNDE) that has its head office at Imperial College. He has published over 170 refereed journal papers and a similar number of conference papers in this field and holds four current patents. He is a director of two spin-out companies set up to exploit technology developed in his research group (Guided ultrasonics, Ltd. and Permasense, Ltd., both of which supply inspection and monitoring equipment to the petrochemical and other industries), and he is a consultant to a variety of industries.

Prof. Cawley is a Fellow of the Royal Academy of Engineering and the Royal Society. 